\title{
Sergio Secondiano Sacchi (Hg.): Multiflter. Mito e memoria del padre nella canzone. Roma: Squi(libri), 2017. ISBN 978-8885571020. 208 Seiten, 2 CDs.
}

Sergio Secondiano Sacchi ist neben Antonio Silva das dienstälteste Mitglied der Leitung des 1974 gegründeten Club Tenco, seit 2017 auch einer der beiden kulturellen Leiter des Clubs. Sacchi ist Architekt und hat einen breiten kulturellen Horizont, nicht nur in Sachen Canzoni. Vor einigen Jahren gründete er - sozusagen als Filiale - den Club Le cose di Amilcare in Barcelona, wo er auch lebt. Seine Kontakte zu Musikern und Künstlern sind vielschichtig, seine Liebe gehört primär dem Autorenlied. Soviel muss man zum Herausgeber wissen, um diese kuriose und vielschichtige Publikation einordnen zu können.

Im Rahmen des Club Tenco hat Sacchi 2017 das Buch Multifilter. Mito e memoria del padre nella canzone herausgegeben, 208 Seiten stark und mit zwei CDs von je 18 Aufnahmen.

In der langen, eher leserunfreundlich strukturierten Einleitung erfahren wir viel über Gott, die Welt, soziologische und psychologische Einblicke in die Kultur Europas und Amerikas, aber relativ wenig über die Kriterien der Auswahl der Lieder und der Begleittexte bzw. den Unterschied zwischen mito und memoria. Auch wäre es durchaus sinnvoll gewesen, zum besseren Verständnis der Anthologie eine Definition dessen zu geben, was der Herausgeber unter Canzone versteht. Von der Vertonung einer Boccaccio-Novelle über spanische Gedichte aus dem 16. Jahrhundert bis zu einer Komposition von Astor Piazzolla ist die Spannweite doch etwas sehr breit angesetzt.

Kürzer, prägnanter und informativer als die Einleitung sind die Texte, welche die 36 Songs begleiten. Allerdings wäre es nützlich gewesen, die AutorInnen auch mit einem minimalen biographischen Index vorzustellen. Wer nicht weiß, dass Antonio Silva der , historische' Präsentator des Club Tenco ist, wird dessen locker eingestreuten Text kaum einordnen können. Es scheint, hier sei wieder einmal ein Buch ohne professionelles Lektorat in Druck gegeben worden.

Diesen redaktionellen Schwächen stehen zwei starke Pluspunkte gegenüber.

Einerseits die zahlreichen Illustrationen, unter anderen von Sacchi selbst, der auch das Cover gestaltet hat. Für die Gestaltung des Bandes zeichnet sein Freund Roberto Molteni, auch er künstlerisch tätig und Mitglied der engeren Leitung des Club Tenco. Außerordentlich sind natürlich die Illustrationen von Sergio Staino, dem Schöpfer des legendären Bobo. Auch er gehört zum engen Dunstkreis des Club Tenco.

Die evidenten Stärken der Publikation sind die insgesamt 36 Lieder der beiden CDs und die dazu gehörenden Texte von diversen AutorInnen. Man mag die nicht deklarierten Auswahlkriterien vermissen, unter dem Strich bleibt viel gute Musik mit durchwegs seltenen 
Aufnahmen, darunter auch künstlerische Highlights, die zum großen Teil eigens für diese Publikation aufgenommen wurden.

Unter den 18 Titeln, die auf CD 1 unter mito präsentiert werden, gibt es echte Trouvaillen. Vittorio De Scalzi, Gründungsmitglied der legendären New Trolls, hat einen der größten Hits der Band, „Quella carezza nella sera“ von 1978, in seinem Home-Studio in Sanremo neu aufgenommen und gleich alle Instrumente selbst gespielt. Illustriert wird dieser berührende Song von Staino und einem Begleittext von Dario Zigiotto, über dessen Identität man gerne etwas erfahren würde. Ein Bijou ist auch „Quand'è dove si va?" von Mimmo Locasciulli und sieben weiteren Musikern, darunter der Jazz-Bassist Greg Cohen. Der Song stammt von Renaud Séchan und wird in der Übersetzung von Sacchi präsentiert. Ein weiterer Höhepunkt ist „La sera che partì mio padre“, ein ebenso berührendes wie engagiertes Lied von Enzo Jannacci, interpretiert von Alessio Lega. Sehr schön ist die Interpretation von Chico Buarque de Holandas „Tanto mar“ durch Luiz Murà (Gesang und Gitarre). Feinsinnig der Song „Più notte di così“ von Mauro Ermanno Giovanardi, der wie Luca Ghielmettis „La faccia del papà - das Bild eines Vaters, welcher der Geburt des eigenen Kindes beiwohnt - einen Blick auf die Vaterrolle der Gegenwart wirft.

Unerträglich hingegen ist die Version des Eröffnungstitels „O mein Papa“ im Arrangement von Gianfrancesco Calabrese. Da wäre eine originale Aufnahme des Schweizer Musicals von 1939 angezeigt gewesen. Unverständlich auch, wie in diesem Kontext kein Song von Fabrizio de André zu finden ist. Keiner hätte wie er dem Kriterium entsprochen, nach dem das Bild des Vaters im Lied den sozialen Wandel der Zeit dokumentiert. Man denke an den „bombarolo“ von „Storia di un impiegato“ (1973) und dessen Zerwürfnis mit dem Vater; das war schon damals mehr als eine einfache Auseinandersetzung mit 1968. Mit dem 68er Mythos setzt sich immerhin im abschließenden Song der CD 1 „Dopo dieci anni“ Ricky Gianco auseinander.

Die zweite CD befasst sich mit der memoria. Hier ist der eindrücklichste Moment „Lengenfeld“, in dem Andrea Satta (Têtes de Bois) die Geschichte des grünen Akkordeons beschreibt, das sein Vater aus dem Konzentrationslager Lengenfeld mitbrachte (auch hier eine magistrale - für einmal bedrückende - vignetta von Staino). Beeindruckend auch „L'ultima riga del libro“ des Scraps Orchestra sowie „Il funerale del clown“ von Yo Yo Mundi. In dieser Sektion fehlen auch die Cantautori-Schwergewichtler Francesco Guccini und Roberto Vecchioni nicht, zumindest sind sie indirekt präsent. Guccinis „Van Loon“ ist ein Porträt seines Vaters Ferruccio, wie wir aus dem Begleittext des Autors selbst entnehmen. Interpretiert wird der Song von Silvia Comes auf Spanisch, leider ein fahler Abglanz der Originalversion. Auf Katalanisch und beeindruckend in Intonation und Empathie interpretiert Joan Isaac Vecchionis „L’uomo che si giocava il cielo a dadi“ („L'home que va jugar el cel als daus"). In den Begleittexten kommen der Vater und der Bruder Vecchionis zu Wort. Warum nicht auch einer seiner Söhne?

Einer der stärksten Momente der CD 2 ist „Parole per Julia“, „Palabras para Julia“ von Juan Goytisolo (Text) und Paco Ibáñez (Musik), übersetzt von Sacchi und meisterhaft interpretiert von Sergio Cammariere, der wie kein zweiter unter den jüngeren Cantautori 
Nostalgie und Gefühle in Musik umsetzen kann. Unter den Cantautori der zweiten Generation finden wir auch Giovanni Block mit „L'aquilone“ und Marco Ongaro mit „La fidanzatina" als positive Entdeckungen.

Etwas überraschend erscheint auf CD 2 auch David Riondinos „Tancredi e Ghismonda“, eine seiner in Liedform interpretierten Novellen aus Boccaccios Decamerone. Das wäre wohl eher unter mito zu erwarten gewesen. Erstaunlich auch die Präsenz von „Adiòs Nonino“ von Astor Piazzolla. Die stupende Version - natürlich ohne Text - durch Gianni Coscia (Akkordeon), Mauro Pagani (Geige) und Gianluigi Trovesi (Klarinette) dient als Vorwand für einen Text zur Rolle des Vaters im Tango. „Adios Nonino“ ist natürlich eine Komposition und kein Song, auch wenn das Thema passt.

Alles in allem bietet die multimediale Publikation sehr viel Positives dank der vielen und mehrheitlich gelungenen Illustrationen, der 36 Einspielungen mit Seltenheitswert und der dazu gehörenden Begleittexte.

Rudi ANKLI (Basel) 